\title{
Gender Disparities in the Relationship between Education and Self-Reported Health Across Cohorts in China
}

\section{CURRENT STATUS: ACCEPTED}

BMC Public Health $\triangle$ BMC Series

Bowen Zhu

Hunan Normal University

zhubowen92@126.comCorresponding Author

ORCiD: https://orcid.org/0000-0003-2350-2735

Yiwan Ye

University of California Davis Department of Sociology

DOI:

$10.21203 / \mathrm{rs} .2 .16306 / \mathrm{v} 1$

\section{SUBJECT AREAS}

Health Policy

KEYWORDS

Education and Health Gap, Gender Disparity, Cohort Effect, Age Vector Model 
Abstract

Background: Variation in the relationship between education and health has been studied intensely over the past few decades. Although there is abundant research on gender disparity and cohort variations in the relationship between education and health, based on samples from the U.S. and Europe, research about China is limited. Giventhe specificsocial changes in China, the study is designed to analyze the gender difference and cohort variations in the education-health relationship. Method: Longitudinal, nationwide data from the Chinese Family Panel Studies from the years 2010 to 2016 are statistically analyzed. Self-reported health is measured by respondents' subjective assessment of their health. The highest level of education earned operationalizes the education measure. Each cohort is defined by a distinct period of social change in China. The age-vector model is used to analyze gender and cohort variations in the association between education and self-rated health.

Results: Men report better health than women, but the relationship between education and health for women is stronger than for men. Educational gaps in self-rated health do not change significantly for cohorts before 1955 and cohorts after 1977, but the gaps become stronger for cohorts between 1956 and 1976. There is gender disparity within the cohort variations in the education-health relationship. For women, the education-health relationship in the 1956-1960, 1967-1976 and 1977-1983 cohorts is significantly stronger than for the 1908-1938 cohort. While not as strong, the education-health relationship remains consistent across all cohorts for men.

Conclusions: The study findings support the resource substitution hypothesis and not the rising importance hypothesis in China. Considering the findings on gender disparity and difference in cohort effects, we discuss the potential influences of the unique social transformation and educational expansion in China.

Full-text

Due to technical limitations, full-text HTML conversion of this manuscript could not be completed. However, the manuscript can be downloaded and accessed as a PDF. 